\title{
DINÂMICA DA COMPOSIÇÃO FLORÍSTICA DE UMA FLORESTA OMBRÓFILA DENSA SECUNDÁRIA, APÓS CORTE DE CIPÓS, RESERVA NATURAL DA COMPANHIA VALE DO RIO DOCE S.A., ESTADO DO ESPÍRITO SANTO, BRASIL ${ }^{1}$
}

\author{
Agostinho Lopes de Souza ${ }^{2}$, Stanley Schettino ${ }^{3}$, Renato Moraes de Jesus ${ }^{4}$ e Antonio Bartolomeu do Vale ${ }^{5}$
}

\begin{abstract}
RESUMO - O objetivo deste trabalho foi analisar a dinâmica da composição florística de uma Floresta Ombrófila Densa secundária, após o corte de cipós. O tratamento silvicultural foi executado com o objetivo de promover o rápido retorno da floresta às suas condições primárias ou, no mínimo, diminuir o intervalo de tempo entre dois ciclos de corte sucessivos. Os dados foram provenientes de um experimento implantado em 1989 e medido bienalmente na Reserva Natural da Companhia Vale do Rio Doce S.A., municípios de Linhares e Jaguaré-ES, Brasil. A amostragem foi sistemática (35 parcelas permanentes retangulares), tendo sido considerados como indivíduos adultos as árvores com $d a p \geq 5,0 \mathrm{~cm}$. Foram avaliados os efeitos da aplicação do tratamento sobre a composição florística e a diversidade de espécies, em cada ocasião de monitoramento. No tocante às espécies arbóreas, o tratamento pouco influenciou a dinâmica da composição florística. Embora a diversidade tenha aumentado (0,55\% pelo índice de Shannon-Weaver), os demais índices apresentaram decréscimo, o que indica que houve elevada taxa de ingresso das espécies preexistentes na área, ou seja, o aumento do número de indivíduos na população está ocorrendo em taxas maiores do que o incremento no número de espécies. Já a estrutura da floresta (estrato arbóreo) foi bastante influenciada, pois os substanciais aumentos em número de árvores e área basal indicam que as taxas de recrutamento e crescimento estão superando as de mortalidade. Da mesma forma, os grupos ecofisiológicos das secundárias tardias e climáxicas foram os mais beneficiados, sendo mais um indicativo de que o tratamento está favorecendo a dinâmica de sucessão secundária.
\end{abstract}

Palavras-chave: Manejo, tratamento silvicultural, composição florística e floresta secundária.

\section{FLORISTIC COMPOSITION DYNAMICS OF A SECONDARY DENSE OMBROPHYLOUS FOREST, AFTER VINE CUTTING, AT THE VALE DO RIO DOCE S.A. NATURAL RESERVE IN ESPÍRITO SANTO, BRAZIL}

\begin{abstract}
The objective of this work was to analyze the floristic composition dynamics of a secondary dense ombrophylous forest, after vine cutting. The silvicultural treatment was applied to obtain a rapid return to its primary conditions, or, at least, to shorten the period of time between two successive cutting cycles. Data from an experiment established in 1989 were collected every two years from the Companhia Vale do Rio Doce S.A. Natural Reserve, in Linhares and Jaguaré, ES, Brazil. Systematic sampling was performed (35 permanent rectangular plots), in which trees with dbh $\geq 5.0 \mathrm{~cm}$ were considered adult individuals. The treatment effects on the floristic composition and species diversity were evaluated at each monitoring period. The treatment had little influence on the floristic composition dynamics of the trees. The diversity had increased $0.55 \%$ by the Shannon-Weaver index, but the other indexes decreased. This indicates that a high rate of ingrowths occurred in the area, or an increase in the number of individuals is occurring at greater rates than the increase in the number of species. However, the
\end{abstract}

1 Recebido para publicação em 29.3.2000.

Aceito para publicação em 6.11.2002.

2 Prof. Titular do Departamento de Engenharia Florestal da Universidade Federal de Viçosa - DEF/UFV, 36570-000 ViçosaMG. ${ }^{3}$ Engenheiro Florestal - M.S. - Cenibra, Governador Valadares-MG. ${ }^{4}$ Engenheiro Florestal, D.S., Vale do Rio Doce S.A., Reserva Florestal de Linhares, Linhares-ES. ${ }^{5}$ Prof. Associado do Departamento de Engenharia Florestal da UFV. 
forest structure (arboreal stratum) was greatly influenced, since the substantial increase in both number of trees and basal area suggest that the rates of recruitment and growth overcome those of mortality. Similarly, the ecophysiological groups late secondary forests and climaxes received the greatest benefits, indicating that this treatment favors the secondary succession dynamics.

Key words: Management, silvicultural treatment, floristic composition, secondary forest.

\section{INTRODUÇÃO}

A atual preocupação com o desenvolvimento sustentável não pode ater-se somente aos recursos florestais nativos, mas deve, principalmente, englobar a proteção ambiental como um todo. Mais do que isto, o desenvolvimento sustentável implica um novo conceito de crescimento econômico, capaz de proporcionar oportunidades justas a toda a população mundial, sem privilegiar pequenos grupos e sem destruir os recursos naturais finitos do mundo, garantindo a sustentabilidade dos recursos naturais renováveis (Pronk \& Haq, 1992).

Nos últimos tempos, têm sido marcantes as pressões que os ecossistemas florestais vêm sofrendo no Brasil e no mundo. O problema é que, como todo recurso natural renovável, seu estoque não é fixo, podendo este tanto crescer quanto decrescer, de onde se conclui que sua dinâmica é bastante particular. Esse recurso aumentará se for permitida a regeneração do estoque e decrescerá se esta não for permitida. Portanto, os conhecimentos do estoque potencial e dos processos da dinâmica de crescimento são fundamentais para a utilização, em bases ecologicamente sustentáveis, dos recursos florestais, juntamente com estudos sobre sua viabilidade técnica e econômica.

O principal objetivo da produção sustentada em ecossistemas florestais, de acordo com Davis (1966), é alcançar o equilíbrio entre o crescimento líquido e a colheita, anualmente ou por períodos maiores. Por outro lado, Barros (1986) afirmou que as análises das relações entre a vegetação e as variáveis ambientais devem informar sobre a estrutura dos povoamentos florestais, com base em suas produções volumétricas e embasadas em sua composição florística, que constituem os reflexos das diferentes interações dos fatores ambientais.

Assim, este estudo foi realizado com o objetivo de, concomitantemente à aplicação de um tratamento silvicultural considerado racional (corte de cipós), obter um rápido retorno da floresta às suas condições primárias ou, no mínimo, diminuir o intervalo de tempo entre dois ciclos de corte sucessivos, bem como analisar seus efeitos sobre a dinâmica da composição florística de uma Floresta Ombrófila Densa secundária.

\section{MATERIAL E MÉTODOS}

\subsection{Descrição da Área Experimental}

O presente estudo foi desenvolvido com dados de um experimento instalado na Reserva Natural da Companhia Vale do Rio Doce S.A., municípios de Linhares e Jaguaré-ES, Brasil, em um fragmento de Floresta Ombrófila Densa secundária, denominado Projeto RFL 120/89, que já foi explorada para extração de madeira, mas que vem sendo mantida sob proteção desde sua aquisição em 1956. A área localiza-se entre as coordenadas geográficas $19^{\circ} 06^{\prime}$ e $19^{\circ} 18^{\prime}$ de latitude sul e $39^{\circ} 45^{\prime}$ e $40^{\circ} 19^{\prime}$ de longitude oeste. A altitude local oscila entre 28 e $65 \mathrm{~m}$ e a área está distante $30 \mathrm{~km}$ do centro de Linhares. O acesso se dá através da BR 101, à altura do km 122, sentido norte (Jesus, 2001).

Segundo a classificação de Köppen, a região apresenta clima quente e úmido, que corresponde ao tipo Aw (tropical úmido), com precipitação pluviométrica média anual de $1.403 \mathrm{~mm}$, temperatura média máxima de $25,2^{\circ} \mathrm{C}$ e mínima de $19,1^{\circ} \mathrm{C}$ e umidade relativa do ar média de 84,3\% (Jesus, 1987). De acordo com a terminologia do Projeto RADAMBRASIL, a vegetação está inserida na "Região da Floresta Ombrófila Densa" (Veloso et al., 1991; IBGE, 1993).

\subsection{Demarcação da Área Experimental e Coleta de Dados}

A área de estudo abrange uma superfície de 48,75 ha. Para demarcação da área amostrada (Figura 1), foi estabelecida uma bordadura de $75 \mathrm{~m}$ em relação à rede viária existente. Para marcação de parcelas, primeiro foram abertas picadas (linhas) paralelas e eqüidistantes 
em $100 \mathrm{~m}$. Em seguida, a cada $50 \mathrm{~m}$ foram estabelecidas 35 parcelas permanentes de 20 x $50 \mathrm{~m}$. Para o levantamento dos indivíduos da regeneração natural (aqueles com dap menor que 5,0 cm), foram sorteadas 17 parcelas dentre as 35 , onde em uma faixa de 2 x $30 \mathrm{~m}$ efetivou-se esse procedimento.

No levantamento dos indivíduos com dap menor que 5,0 cm, foram registrados: linha - número da linha; parcela - número da parcela; porte - porte ou forma do indivíduo (Quadro 1); classe de regeneração (Quadro 2); cap (circunferência à altura do peito) - somente para a classe 3 de regeneração; forma de regeneração: $S=$ sementes e $B=$ brotação; e $n=$ número de indivíduos.

O experimento foi instalado em março de 1989. Nessa ocasião, foi executado o corte de todos os cipós existentes e, em seguida, foi efetuado o primeiro levantamento de dados. A partir daí, os levantamentos subseqüentes foram efetuados bienalmente, no mês de março, ou seja, em 1991, 1993, 1995 e 1997.

Quadro 1 - Porte dos indivíduos da regeneração (dap < $5,0 \mathrm{~cm}$ ) amostrados na Reserva Natural da Companhia Vale do Rio Doce S.A., municípios de Linhares e de Jaguaré-ES, Brasil

Table 1-Size of the individuals of the natural regeneration $(\boldsymbol{d b h}<5.0 \mathrm{~cm})$, sampled at the Reserva Natural da Companhia Vale do Rio Doce S.A., Linhares and Jaguaré-ES, Brazil

\begin{tabular}{|c|c|}
\hline Classe & Porte do Indivíduo \\
\hline 1 & Arbóreo \\
2 & Arbustivo \\
3 & Cipó \\
4 & Palmeira \\
5 & Herbáceo \\
\hline
\end{tabular}

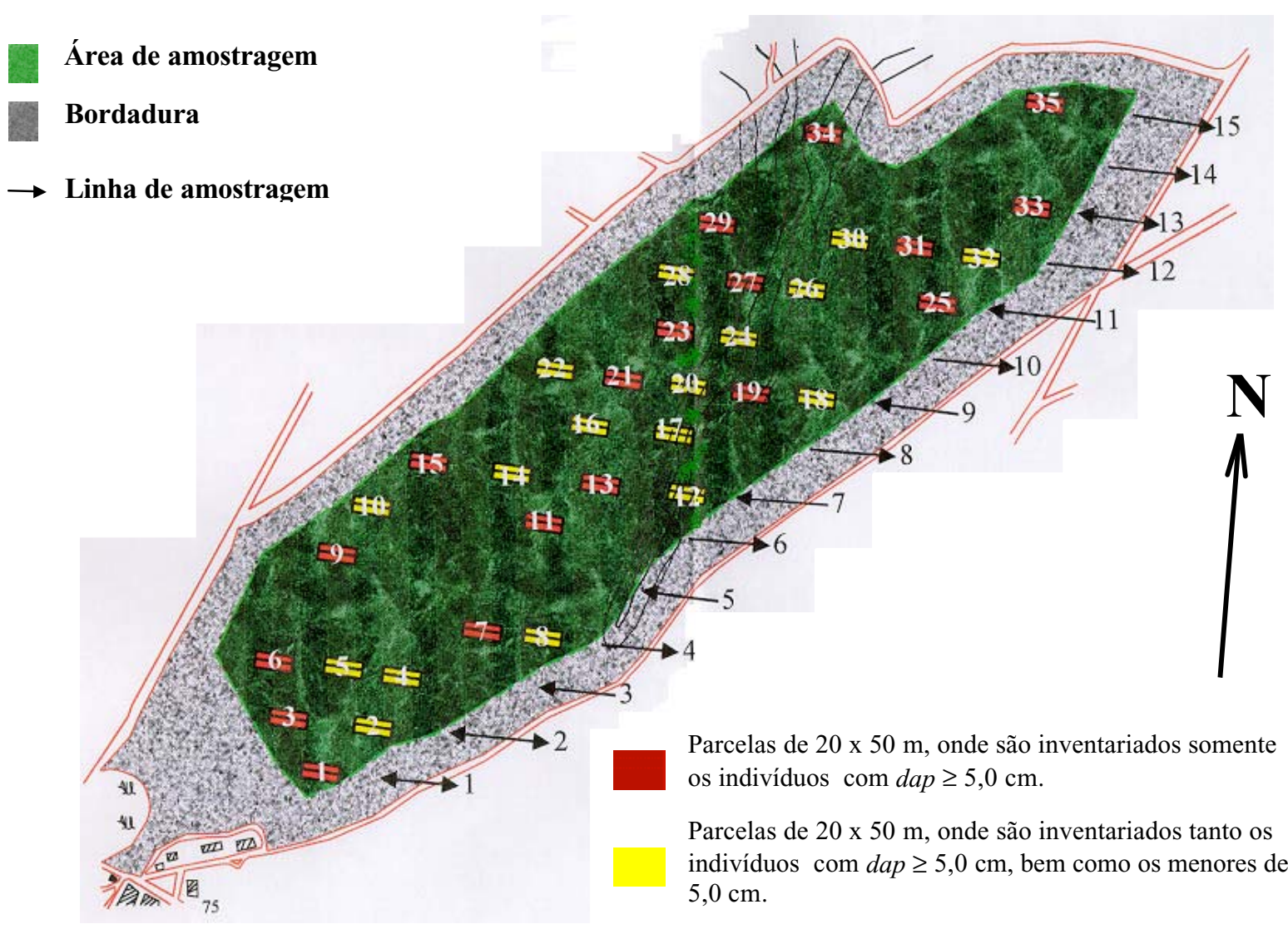

Figura 1 - Distribuição das parcelas na área de estudo, Reserva Natural da Companhia Vale do Rio Doce S.A., municípios de Linhares e Jaguaré, ES, Brasil.

Figure 1 -Study area plot distribution, Reserva Natural da Companhia Vale do Rio Doce S.A., Linhares and Jaguaré, ES, Brazil. 
Quadro 2 - Classes de regeneração natural (CTRN) amostradas na Reserva Natural da Companhia Vale do Rio Doce S.A., municípios de Linhares e de Jaguaré-ES, Brasil

Table 2 -Classes of natural regeneration (CTRN) sampled at the Reserva Natural da Companhia Vale do Rio Doce S.A., Linhares and Jaguaré-ES, Brazil

\begin{tabular}{|c|c|c|c|}
\hline CTRN & Intervalo & cap & Contagem \\
\hline 1 & $H<0,30 \mathrm{~m}$ & Não & Sim \\
2 & $H \geq 0,30 \mathrm{~m} \mathrm{e} \operatorname{cap}<8,0 \mathrm{~cm}$ & Não & Sim \\
3 & $8,0 \mathrm{~cm} \leq \operatorname{cap}<15,5 \mathrm{~cm}$ & Sim & Não \\
\hline
\end{tabular}

\subsection{Análise dos Dados}

Com os dados básicos das amostragens efetuadas nas ocasiões sucessivas, foram obtidas as estimativas dos parâmetros florísticos, cujas análises contemplaram as listagens das espécies arbóreas ocorrentes na área de estudo, preparadas por Peixoto (1998), nas quais constam, inclusive, os grupos ecofisiológicos e a diversidade das espécies. Foram utilizados os seguintes índices: índice de diversidade ecológica de Shannon-Weaver $\left(H^{\prime}\right)$, índice de diversidade ecológica de Simpson $(C)$, equabilidade de Pielou $(J)$ e coeficiente de mistura de Jentsch $(Q M)$.

\section{RESULTADOS E DISCUSSÃO}

\subsection{Composição Florística}

Observou-se, para o conjunto das cinco medições, a ocorrência de árvores de 344 espécies, pertencentes a 57 famílias botânicas.

Com relação ao número de espécies por famílias, apesar de terem sido verificadas algumas alterações, o comportamento não se modificou demasiadamente durante o período de monitoramento (Quadro 3). Assim, ao longo do período (oito anos), as seguintes famílias merecem destaque sob esse aspecto: Myrtaceae (45 espécies), Sapotaceae (27), Leguminosae Caesalpinioideae (25), Leguminosae Faboideae (17), Leguminosae Mimosoideae (14), Lauraceae (14), Moraceae (12), Euphorbiaceae (11), Flacourtiaceae (11) e Chrysobalanaceae (11). Estas dez famílias (17,54\% do total) abrangem 54,36\% dos indivíduos amostrados em 1997. Estes resultados concordam com as argumentações de Richards (1957), citado por Mariscal Flores (1993), que relatou ser comum, em florestas tropicais pluviais, o fato de poucas famílias deterem o maior número de indivíduos, dizendo que este predomínio numérico em uma mesma família botânica expressa a dominância da família na área. No Brasil, dominam as Leguminosas como um todo (Silva \& Leitão Filho, 1982).

As famílias Bixaceae, Caryocaraceae, Convolvulaceae, Dichapetalacaceae, Ebenaceae, Humiriaceae, Icacinaceae, Lacistemaceae, Malvaceae, Polygonaceae, Polygalaceae, Symplocaceae, Trigoniaceae, Ulmaceae e Violaceae apresentaram-se com uma única espécie cada. Em termos de gêneros, destacam-se: Eugenia (18 espécies), Pouteria (14), Ocotea (11), Inga (8), Casearia (7), Marlierea (7), Plinia (6), Trichilia (6), Swartzia (6) e Myrcia (5). No outro extremo, ou seja, com apenas uma espécie cada, foram amostrados 131 gêneros. Além disto, observou-se que 132 espécies ocorrem exclusivamente no estrato arbóreo da floresta estudada.

\subsection{Dinâmica da Composição Florística}

Como o tratamento não previa colheitas de madeira, nenhuma espécie arbórea foi suprimida em decorrência de cortes. Apesar do pequeno intervalo de tempo (19891997) entre a primeira e a última medição e do estádio avançado de sucessão em que se encontra a floresta, podese afirmar que, no tocante às espécies arbóreas com dap $\geq 5,0 \mathrm{~cm}$, o controle da população de cipós influenciou a dinâmica da composição florística. Desta forma, em 1989 (primeira medição, logo após a aplicação do tratamento) foram amostradas 321 espécies, pertencentes a 56 famílias botânicas (Figura 2). Em 1997, ou seja, oito anos após a aplicação do tratamento, verificou-se a presença de 340 espécies, pertencentes a 57 famílias botânicas, o que representa incremento de 5,92 e 1,78\% em número de espécies e famílias, respectivamente. 
Quadro 3 - Riqueza de espécies por família e ano de medição, em ordem decrescente de número de espécies, em 1989. Reserva Natural da Companhia Vale do Rio Doce S.A., municípios de Linhares e Jaguaré-ES, Brasil

Table 3 - Wide range of species by family and year of measurement in decreasing order of number of species, in 1989. Companhia Vale do Rio Doce S.A., Natural Reserve in Linhares and Jaguaré, ES, Brazil

\begin{tabular}{|c|c|c|c|c|c|}
\hline \multirow{2}{*}{ Família } & \multicolumn{5}{|c|}{ Ano de Medição } \\
\hline & 1989 & 1991 & 1993 & 1995 & 1997 \\
\hline Myrtaceae & 40 & 42 & 44 & 44 & 45 \\
\hline Sapotaceae & 26 & 26 & 26 & 27 & 27 \\
\hline Leguminosae Caesalpinioideae & 24 & 25 & 25 & 25 & 25 \\
\hline Leguminosae Faboideae & 17 & 17 & 17 & 17 & 17 \\
\hline Lauraceae & 13 & 14 & 14 & 14 & 14 \\
\hline Leguminosae Mimosoideae & 12 & 12 & 14 & 14 & 14 \\
\hline Moraceae & 12 & 12 & 12 & 12 & 12 \\
\hline Euphorbiaceae & 11 & 11 & 11 & 11 & 11 \\
\hline Flacourtiaceae & 11 & 11 & 11 & 11 & 11 \\
\hline Rubiaceae & 11 & 11 & 11 & 10 & 10 \\
\hline Chrysobalanaceae & 10 & 11 & 11 & 11 & 11 \\
\hline Annonaceae & 8 & 8 & 9 & 8 & 9 \\
\hline Lecythidaceae & 8 & 8 & 8 & 8 & 8 \\
\hline Meliaceae & 8 & 8 & 9 & 9 & 9 \\
\hline Anacardiaceae & 7 & 7 & 7 & 7 & 7 \\
\hline Rutaceae & 7 & 7 & 7 & 7 & 8 \\
\hline Apocynaceae & 6 & 6 & 6 & 6 & 6 \\
\hline Bignoniaceae & 6 & 6 & 7 & 7 & 7 \\
\hline Sapindaceae & 6 & 6 & 6 & 6 & 6 \\
\hline Bombacaceae & 5 & 5 & 5 & 5 & 5 \\
\hline Combretaceae & 5 & 5 & 5 & 5 & 5 \\
\hline Melastomataceae & 5 & 5 & 4 & 4 & 4 \\
\hline Olacaceae & 4 & 4 & 4 & 4 & 4 \\
\hline Verbenaceae & 4 & 4 & 4 & 4 & 4 \\
\hline Boraginaceae & 3 & 3 & 3 & 3 & 3 \\
\hline Burseraceae & 3 & 3 & 4 & 4 & 4 \\
\hline Monimiaceae & 3 & 3 & 3 & 3 & 3 \\
\hline Sterculiaceae & 3 & 3 & 3 & 3 & 3 \\
\hline Tiliaceae & 3 & 3 & 3 & 3 & 3 \\
\hline Vochysiaceae & 3 & 3 & 3 & 3 & 3 \\
\hline Araliaceae & 2 & 2 & 2 & 2 & 2 \\
\hline Caricaceae & 2 & 2 & 2 & 2 & 2 \\
\hline Cecropiaceae & 2 & 2 & 2 & 2 & 2 \\
\hline Celastraceae & 2 & 2 & 2 & 2 & 2 \\
\hline Clusiaceae & 2 & 3 & 3 & 3 & 4 \\
\hline Elaeocarpaceae & 2 & 2 & 2 & 2 & 2 \\
\hline Malpighiaceae & 2 & 2 & 2 & 2 & 2 \\
\hline Myristicaceae & 2 & 2 & 2 & 2 & 2 \\
\hline Rhamnaceae & 2 & 2 & 2 & 2 & 2 \\
\hline Simaroubaceae & 2 & 2 & 2 & 2 & 2 \\
\hline Solanaceae & 2 & 2 & 2 & 2 & 2 \\
\hline Bixaceae & 1 & 1 & 1 & 1 & 1 \\
\hline Caryocaraceae & 1 & 1 & 1 & 1 & 1 \\
\hline Convolvulaceae & 1 & 1 & 1 & 1 & 1 \\
\hline Dichapetalaceae & 1 & 1 & 1 & 1 & 1 \\
\hline Ebenaceae & 1 & 1 & 1 & 1 & 1 \\
\hline Humiriaceae & 1 & 1 & 1 & 1 & 1 \\
\hline Icacinaceae & 1 & 1 & 1 & 1 & 1 \\
\hline Lacistemaceae & 1 & 1 & 1 & 1 & 1 \\
\hline Malvaceae & 1 & 1 & 1 & 1 & 1 \\
\hline Nyctaginaceae & 1 & 1 & 2 & 2 & 3 \\
\hline Polygalaceae & 1 & 1 & 1 & 1 & 1 \\
\hline Polygonaceae & 1 & 1 & 1 & 1 & 1 \\
\hline Trigoniaceae & 1 & 1 & 1 & 1 & 1 \\
\hline Ulmaceae & 1 & 1 & 1 & 1 & 1 \\
\hline Violaceae & 1 & 1 & 1 & 1 & 1 \\
\hline Symplocaceae & & & & & 1 \\
\hline Total & 321 & 327 & 335 & 334 & 340 \\
\hline
\end{tabular}




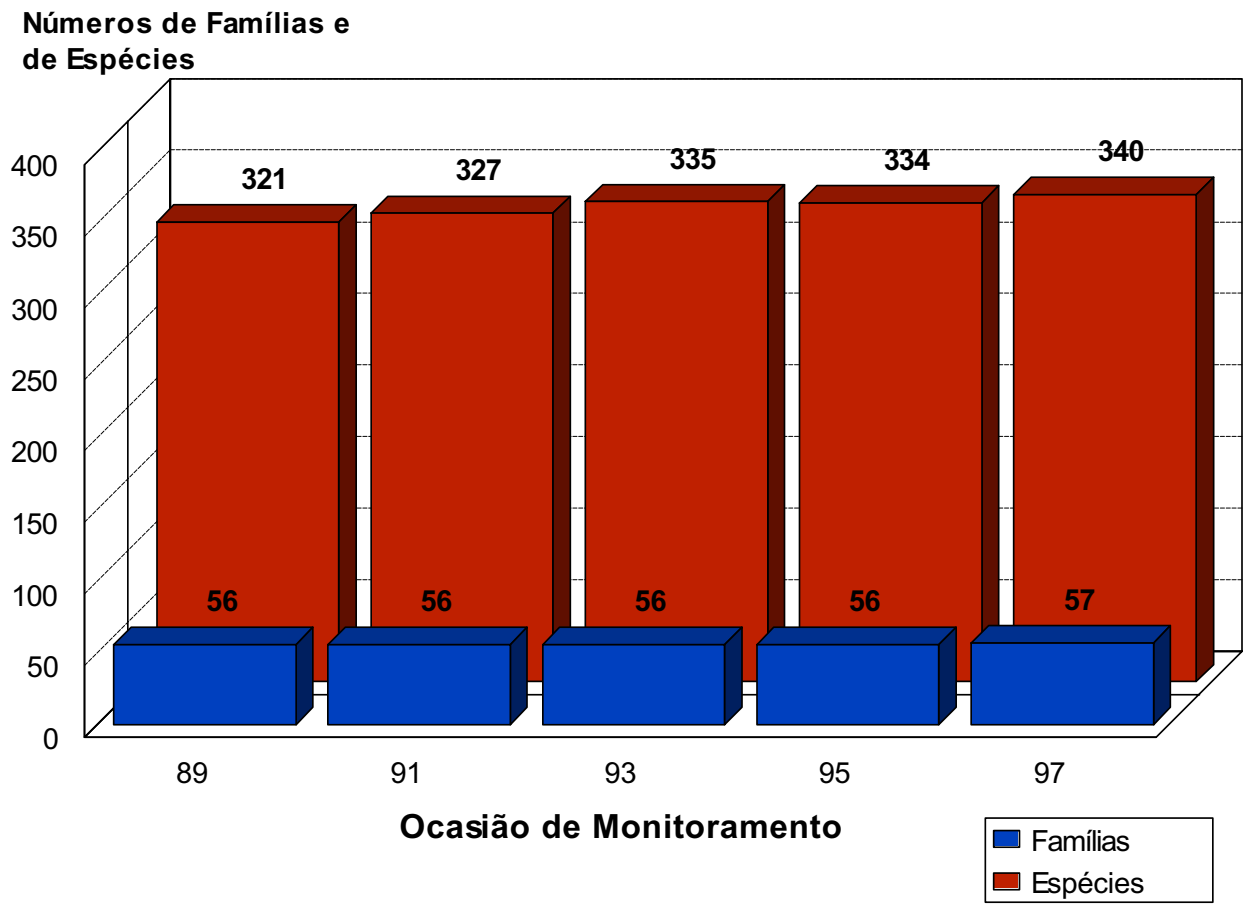

Figura 2 - Número de famílias e de espécies amostradas em cada ocasião de monitoramento - Reserva Natural da Companhia Vale do Rio Doce S.A., municípios de Linhares e Jaguaré-ES, Brasil.

Figure 2 - Number of families and species sampled at each monitoring period - Companhia Vale do Rio Doce S.A., Natural Reserve in Linhares and Jaguaré, ES, Brazil.

Em 1991 (dois anos após a medição inicial), foram amostradas seis novas espécies, quais sejam: Eugenia sp. (secundária tardia), Hirtella insignis Briq. \& Prance (secundária tardia), Hymenaea courbaril L. (clímax), Myrcia isaiana G.M. Barroso \& Peixoto (secundária tardia), Ocotea glauca Mez (secundária tardia) e Vismia aff. martiana H.G. Reich (pioneira). Nenhuma das espécies amostradas na primeira ocasião desapareceu após o intervalo de monitoramento de dois anos.

Em 1993 (após quatro anos), nove novas espécies foram amostradas em relação à medição de 1991: Annona acutiflora Mart. (secundária tardia), Cabralea canjerana (Vell.) Mart. (clímax), Eugenia cuspidata Berg (clímax), Guapira noxia (Netto) Lundell (secundária inicial), Inga hispida Schott ex Benth. (clímax), Inga cordistipula Mart. (secundária tardia), Marlierea acuminatissima (Berg) C.D. Legrand (secundária tardia), Protium heptaphyllum March. (secundária inicial) e Tabebuia obtusifolia Bureau (secundária inicial). Contudo, neste intervalo desapareceu a espécie Miconia mirabilis (Aubl.) L.O. Willians (secundária inicial).

R. Árvore, Viçosa-MG, v. 26, n.5, p.549-558, 2002
Decorridos seis anos da medição inicial, ou seja, em 1995, surgiu a espécie Lucuma butyrocarpa Kuhlm. (clímax) e desapareceu a espécie Tocoyena brasiliensis Mart. (secundária tardia). Ao final de oito anos, ou seja, em 1997, surgiram Annona cacans Warm. (secundária inicial), Galipea jasminifolia Engl. (clímax), Kielmeyera occhioniana Saddi (secundária inicial), Marlierea sucrei G.M. Barroso \& Peixoto (secundária inicial), Pisonia ambigua Heimerl (secundária inicial), Plinia rivularis (Cambess.) A.D. Rotman (secundária tardia) e Symplocos sp. (secundária inicial), perfazendo sete novas espécies. No mesmo período, a espécie Calyptranthes lucida Mart. ex DC. (clímax) desapareceu.

Vale ressaltar que a espécie Annona acutiflora (secundária tardia), que surgiu na florística em 1993, veio a desaparecer na medição subseqüente, ou seja, em 1995.

No tocante aos grupos ecofisiológicos e considerando o período de oito anos, constata-se que ingressaram 23 novas espécies, sendo uma pioneira, oito secundárias iniciais, oito secundárias tardias e seis clímax (Figura 3). Nesse mesmo período desapareceram quatro espécies, 
sendo uma secundária inicial, duas secundárias tardias e uma clímax. A saída de duas espécies secundárias tardias e de uma espécie-clímax e o surgimento de espécies pioneiras e secundárias iniciais se devem, provavelmente, às dinâmicas de sucessão em clareiras formadas pela morte natural de grandes árvores, pertencentes aos grupos ecofisiológicos representativos dos estádios mais avançados de sucessão florestal, conforme descrito por Jardim et al. (1993).

\subsection{Diversidade de Espécies}

Os valores dos índices de diversidade de espécies arbóreas, por ocasião de medição, estão apresentados no Quadro 4, e os valores porcentuais de variação no intervalo de tempo entre as medições encontram-se no Quadro 5.

De acordo com o índice de diversidade de ShannonWeaver $\left(H^{\prime}\right)$, a equabilidade de Pielou $(J)$ e o índice de diversidade ecológica de Simpson $(C)$, a área apresentou alta diversidade de espécies arbóreas, sendo $H^{\prime}$ (em todas as medições) bastante próximo ao valor encontrado por
López (1996) em uma área adjacente à do estudo, que foi de 5,11, e um tanto superior a alguns índices estimados em outros trabalhos executados em tipologias florestais pertencentes à área de domínio da Floresta Atlântica, como: $H^{\prime}=4,360$ (SILVA, 1989); $H^{\prime}=3,809$ (Mariscal Flores, 1993); $H^{\prime}=3,090$ (Calegário, 1993); $H^{\prime}=3,967$ (Almeida, 1996); $H^{\prime}=4,710$ (Souza et al., 1998a); $H^{\prime}=$ 4,640 (Souza et al., 1998b); e $H^{\prime}=3,561$ (Souza et al., 1997).

Como pode ser observado no Quadro 5, foram verificadas variações em todos os índices, ao longo dos intervalos de medições. Entretanto, para o coeficiente de mistura de Jentsch (QM) verificou-se o maior porcentual de variação (-18,30\% ao longo de oito anos), o que significa que, em média, a relação entre o número de espécies e o número de indivíduos passou de 1:11, em 1989, para 1:13, no final do período de medições, ou seja, em 1997. Numa análise mais parcimoniosa e levando em consideração o índice de diversidade de ShannonWeaver $\left(H^{\prime}\right)$, que variou em $0,55 \%$ no mesmo período (Quadro 5, Figura 4), pode-se afirmar que a taxa de ingresso das espécies preexistentes na área foi muito

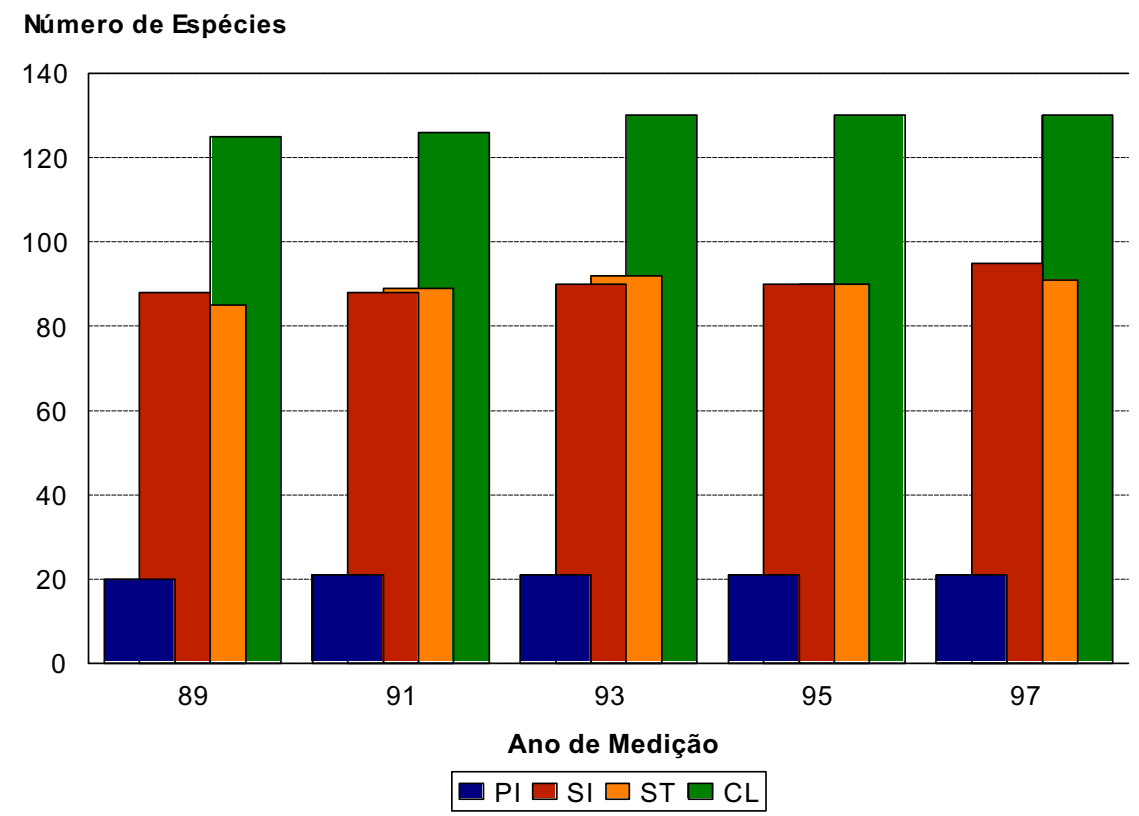

Figura 3 - Evolução do número de espécies, por grupos ecofisiológicos, ao longo do intervalo de monitoramento - Reserva Natural da Companhia Vale do Rio Doce S.A., municípios de Linhares e Jaguaré-ES, Brasil, em que PI = pioneira, $S I=$ secundária inicial $S T=$ secundária tardia e $C L=$ clímax.

Figura 3 -Evolution of the number of species by ecophysiologic group, during the monitoring period - Companhia Vale do Rio Doce S.A. Natural Reserve in Linhares and Jaguaré, ES, Brazil, where: $\boldsymbol{P I}=$ pioneers $\mathbf{S I}=$ initial secondary $\mathbf{S T}=$ late secondary; and $\boldsymbol{C L}=$ climax. 
elevada, conforme ilustrado na Figura 3, em detrimento do recrutamento de novas espécies. Este fato é corroborado pelo índice de diversidade ecológica de Simpson (C), que passou de 0,0117, em 1989 (valor considerado baixo), para 0,0109, em 1997, ou seja, uma redução de $6,84 \%$, evidenciando que o aumento do número de indivíduos na população está ocorrendo a taxas maiores que a do incremento no número de espécies.

Quadro 4 - Índices de diversidade de espécies arbóreas, por ocasião de medição, encontrados na Reserva Natural da Companhia Vale do Rio Doce S.A., municípios de Linhares e Jaguaré-ES, Brasil

Table 4-Diversity indexes of species sampled within natural regeneration, at the Companhia Vale do Rio Doce S.A. Natural Reserve in Linhares and Jaguaré, ES, Brazil

\begin{tabular}{|l|c|c|c|c|c|}
\hline \multicolumn{1}{|c|}{ Índice } & 1989 & 1991 & 1993 & 1995 & 1997 \\
\hline Coeficiente de mistura de Jenstcht (QM) & 0,0951 & 0,0911 & 0,0840 & 0,0822 & 0,0777 \\
Índice de diversidade de Shannon Weaver (H') & 5,0305 & 5,0386 & 5,0477 & 5,0505 & 5,0581 \\
Índice de diversidade ecológica de Simpson (C)(1-C) & 0,0117 & 0,0115 & 0,0113 & 0,0111 & 0,0109 \\
Equabilidade de Pielou (J') & 0,8716 & 0,8702 & 0,8682 & 0,8691 & 0,8678 \\
\hline
\end{tabular}

Quadro 5 - Variações porcentuais dos índices de diversidade florística ao longo dos intervalos de medição - Reserva Natural da Companhia Vale do Rio Doce S.A., municípios de Linhares e Jaguaré, ES, Brasil

Table 5 - Percentage variations of the indexes of floristic diversity, during the monitoring periods - Companhia Vale do Rio Doce S.A. Natural Reserve in Linhares and Jaguaré, ES, Brazil

\begin{tabular}{|c|c|c|c|c|c|}
\hline \multirow{2}{*}{ Índice } & \multicolumn{5}{|c|}{ Intervalo de Medição } \\
\hline & $89 / 91$ & $91 / 93$ & $93 / 95$ & $95 / 97$ & $89 / 97$ \\
\hline QM & $-4,21 \%$ & $-7,79 \%$ & $-2,14 \%$ & $-5,47 \%$ & $-18,30 \%$ \\
\hline $\mathrm{H}^{\prime}$ & $+0,16 \%$ & $+0,18 \%$ & $+0,05 \%$ & $+0,15 \%$ & $+0,55 \%$ \\
\hline $\mathrm{C}$ & $-1,71 \%$ & $-1,74 \%$ & $-1,77 \%$ & $-1,80 \%$ & $-6,84 \%$ \\
\hline $\mathrm{J}^{\prime}$ & $-0,16 \%$ & $-0,23 \%$ & $+0,10 \%$ & $-0,15 \%$ & $-0,44 \%$ \\
\hline $\mathrm{H}$ & $+1,83 \%$ & $+4,47 \%$ & $+2,31 \%$ & $+1,33 \%$ & $+9,61 \%$ \\
\hline
\end{tabular}

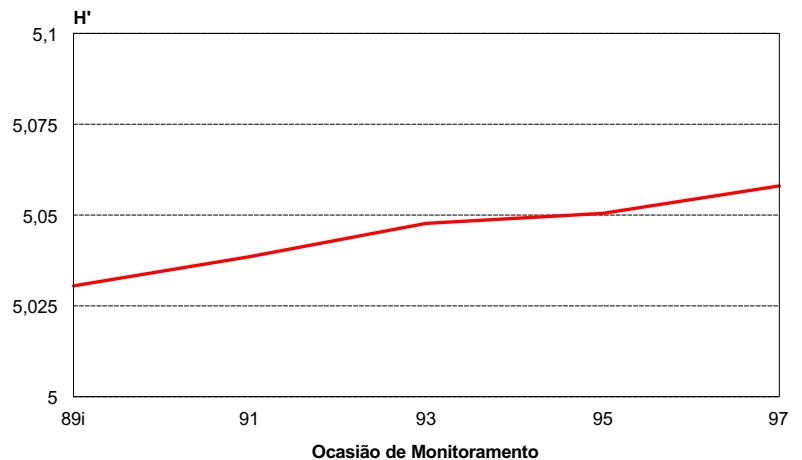

Figura 4 - Comportamento do índice de diversidade de Shannon-Weaver $\left(H^{\prime}\right)$ ao longo das ocasiões de monitoramento, considerando apenas os indivíduos adultos, ou seja, com dap $\geq 5,0 \mathrm{~cm}$ - Reserva Natural da Companhia Vale do Rio Doce S.A., municípios de Linhares e Jaguaré-ES, Brasil.

Figure 4 - Behavior of the Shannon-Weaver Diversity Index (H'), during the monitoring periods, using adult individuals only, i.e., those with $\boldsymbol{d b h} \geq 5.0 \mathrm{~cm}$ Companhia Vale do Rio Doce S.A. Natural Reserve in Linhares and Jaguaré, ES, Brazil.

R. Árvore, Viçosa-MG, v. 26, n.5, p.549-558, 2002

\section{CONCLUSÕES}

Os resultados obtidos no presente trabalho permitiram as seguintes conclusões:

a) Devido ao avançado estádio de sucessão em que se encontra a floresta, ao pequeno intervalo de tempo entre a primeira e a última medição e ao fato de o tratamento não prescrever colheitas de madeira, a dinâmica da composição florística dos indivíduos arbóreos adultos $(d a p \geq 5,0 \mathrm{~cm})$ foi pouco influenciada.

b) O aumento do número de indivíduos na população está ocorrendo a taxas maiores que a do incremento no número de espécies, fato verificado pela diminuição do valor do coeficiente de mistura de Jentsch, pela reduzida elevação do índice de diversidade de Shannon-Weaver e pela diminuição observada no índice de diversidade ecológica de Simpson, ou seja, 
embora tenha sido observado aumento de diversidade, este não foi proporcional ao aumento observado na densidade.

c) Os maiores aumentos em número de árvores ocorreram entre 1991 e 1993, ou seja, cerca de quatro anos após a aplicação do tratamento. Após esse período as taxas de crescimento passaram a decrescer, portanto é o período em que são verificados os maiores efeitos da aplicação do tratamento.

d) O corte de cipós é um tratamento silvicultural que pode proporcionar mais rapidamente o retorno de uma floresta secundária às suas condições originais, uma vez que seus efeitos também são verficados sobre a composição florística, contribuindo, assim, para os processos da dinâmica de sucessão secundária.

e) O corte de cipós, técnica notadamente importante, não deve ser considerado isoladamente em um sistema de manejo. A associação com outros tratamentos silviculturais é desejável, pois, da mesma forma, esperase a obtenção de resultados positivos, o que contribui de maneira mais eficiente para a sustentabilidade do sistema de manejo.

\section{REFERÊNCIAS BIBLIOGRÁFICAS}

ALMEIDA, D. S. Manejo para a conservação da diversidade de espécies arbóreas em fragmentos de Floresta Atlântica (Campus da UFJF - Juiz de Fora Minas Gerais). Viçosa: UFV, 1996. 105 p. Dissertação (Mestrado em Ciência Florestal) - Universidade Federal de Viçosa, 1996.

BARROS, P. L. C. Estudo fitossociológico de uma floresta tropical úmida no planalto de Curuá-Una, Amazônia Brasileira. Curitiba: UFPR, 1986. 147 p. Tese (Doutorado em Ciência Florestal) - Universidade Federal do Paraná, 1986.

\section{CALEGÁRIO, N. Parâmetros florísticos e}

fitossociológicos da regeneração natural de espécies arbóreas nativas no sub-bosque de povoamentos de Eucalyptus, no município de Belo Oriente/MG. Viçosa: UFV, 1993, 114 p. Dissertação (Mestrado em Ciência Florestal) - Universidade Federal de Viçosa, 1993.

DAVIS, R. P. Forest management: regulation and valuation. 2.ed. New York: McGraw-Hill, 1966. 519 p.
FUNDAÇÃO INSTITUTO BRASILEIRO DE GEOGRAFIA E ESTATÍSTICA - IBGE. Mapa da vegetação do Brasil. Brasília: IBGE, 1993. 1 p.

JARDIM, F. C. S.; VOLPATO, M. M. L.; SOUZA, A. L. Dinâmica de sucessão natural em clareiras de florestas tropicais. Viçosa: Sociedade de Investigações Florestais, 1993. 60 p. (Documento SIF, 10).

JESUS, R. M. A Reserva Florestal da Companhia Vale do Rio Doce, Linhares-ES: a experiência da CVRD. In.: SEMINÁRIO SOBRE DESENVOLVIMENTO ECONÔMICO E IMPACTO AMBIENTAL EM ÁREA DE TRÓPICO ÚMIDO BRASILEIRO, 1987, Belém. Anais... Belém: Companhia Vale do Rio Doce, 1987. 333 p.

JESUS, R. M. Manejo florestal: impactos da exploração na estrutura da floresta e sua sustentabilidade econômica. Campinas: UNICAMP, 2001. 244 p. Tese (Doutorado em Ecologia) - Universidade de Campinas, 2001.

LÓPEZ, J. A. Caracterização fitossociológica e avaliação econômica de um fragmento de Mata Atlântica secundária, município de Linhares-ES. Viçosa: Universidade Federal de Viçosa, 1996. 71 p. Dissertação (Mestrado em Ciência Florestal) - Universidade Federal de Viçosa, 1996.

MARISCAL FLORES, E. J. Potencial produtivo e alternativas de manejo sustentável de um fragmento de Mata Atlântica secundária, município de Viçosa, Minas Gerais. Viçosa: Universidade Federal de Viçosa, 1993. 165 p. Dissertação (Mestrado em Ciência Florestal) Universidade Federal de Viçosa, 1993.

PEIXOTO, A. L. Análise da Reserva: fatores bióticos. In: Plano Diretor de Uso da Reserva Florestal de Linhares. Vitória: 1998. (Terceiro Relatório Técnico Parcial)

PRONK, J.; HAQ, M. The Hague Report - Sustainable development: from concept to action. New York: UNDP United Nations Development Programme, 1992. 32 p.

SILVA, A. F. Composição florística e estrutura fitossociológica do estrato arbóreo da Reserva Florestal Professor Augusto Ruschi, São José dos Campos, SP. Campinas: UNICAMP, 1989. 163 p. Tese (Doutorado em Ciência Florestal) - Universidade de Campinas, 1989.

SILVA, A. F.; LEITÃO FILHO, H. F. Composição florística e estrutura de um trecho de Mata Atlântica de encosta no município de Ubatuba (São Paulo, Brasil). Revista Brasileira de Botânica, v. 5, p. 43-52, 1982.

R. Árvore, Viçosa-MG, v. 26, n.5, p.549-558, 2002 
SOUZA, A. L. et al. Avaliação de impactos das operações de colheita de madeira e de tratamentos silviculturais num projeto de manejo sustentável. Viçosa: Universidade Federal de Viçosa, 1997. (Relatório de Pesquisa - CNPq).

SOUZA, A. L.; MEIRA NETO, J. A. A.; SCHETTINO, S. Avaliação florística, fitossociológica e paramétrica de um fragmeno de floresta atlântica secundária, município de Caravelas, Bahia. Viçosa: Sociedade de Investigações Florestais, 1998a. 117 p. (Documento SIF, 19).
SOUZA, A. L.; MEIRA NETO, J. A. A.; SCHETTINO, S. Avaliação florística, fitossociológica e paramétrica de um fragmeno de floresta atlântica secundária, município de Pedro Canário, Espírito Santo. Viçosa: Sociedade de Investigações Florestais, 1998b. 121 p. (Documento SIF, 18).

VELOSO, H. P.; RANGEL FILHO, A. L. R.; SILVA, J. C. A. Classificação da vegetação brasileira adaptada a um sistema universal. Rio de Janeiro: IBGE, 1991. 123 p. 